\title{
Removal of FIB Damage using Flash Electropolishing for Artifact-free TEM Foils
}

\author{
Alan Schemer-Kohrn ${ }^{1 *}$, Mychailo B. Toloczko ${ }^{1}$, Yuanyuan Zhu ${ }^{1,2}$, Jing Wang ${ }^{1}$, Danny J. Edwards ${ }^{1}$ \\ 1. Reactor Materials and Mechanical Design Group, Nuclear Sciences Division, Pacific Northwest \\ National Laboratory, Richland, WA 99352, USA \\ 2. Department of Materials Science and Engineering, Institute of Materials Science, University of \\ Connecticut, Storrs, CT 06269, USA \\ * Corresponding author: alan.schemerkohrn@pnnl.gov
}

To prepare samples from both unirradiated and neutron/ion irradiated HT-9, a 12Cr-1Mo-VW ferritic alloy, we utilized focused Ga ion beam (FIB) milling. As stated by Aitkaliyeva [1], the introduction of FIB for the preparation of TEM samples from highly radioactive materials produces samples of a minimal volume of material, yielding TEM or APT samples that are essentially non-detectable compared to background radiation. Unfortunately, FIB milling produced a high density of small defect clusters and dislocation loops up to few nanometers in size, obscuring the real defect structure produced during neutron and/or ion irradiation. This is further complicated by a surface reconstruction artefact [2] that produces interference moiré fringes under strong diffraction conditions. Attempts to use successively lower accelerating voltage steps $(30,15,5 \mathrm{keV})$ during thinning followed by a low-energy $2 \mathrm{keV}$ Ga beam to clean up the surface did little to remove either of these artefacts. This demonstrated a need to find a different approach that can consistently remove the artificial defects produced by FIB milling. We followed the flash electropolishing approach first reported by Huang et al [3], and report here our success in utilizing flash electropolishing to produce FIB lamella from HT-9 that are completely free of both the small "black spot" damage and FIB milling induced dislocation loops, as well as the restructured sample surface.

Figure 1 shows the simple setup of the flash electropolishing apparatus developed in house. It consists of a Pyrex beaker sitting in a glycol cooling bath, utilizes a special in-house built relay timer with a time range from $1 \mathrm{~ms}$ to 9.999 seconds, a 30-volt power supply and a digital multimeter to measure the current. A stainless steel sheet covers part of wall inside the beaker and serves as the cathode. A typical starting sample is a 250-300 nm thick FIB lamella mounted to an Au grid. This loaded grid is held by a self-closing Au-coated tweezer and dipped inside the beaker containing a 4\%perhcloric/ethanol electrolyte cooled to $-50^{\circ} \mathrm{C}$. The samples were flash polished from $20-50 \mathrm{~ms}$ using a voltage of 12 to 13 volts, yielding a removal rate of $\sim 3.5 \mathrm{~nm} / \mathrm{ms}$ for HT-9. Repeated steps were applied until perforation, monitored by an optical microscope with a 1000x eyepiece. Experience with numerous samples yielded an eventual success rate of nearly $100 \%$.

TEM/STEM bright field images of the as-FIB sample (Figure $2 \mathrm{a}$ and $2 \mathrm{~b}$ ) are compared to the those from the final flash polished sample (Figure $2 \mathrm{c}$ and $2 \mathrm{~d}$ ). The as-FIB sample contains a high density of black spot damage and moiré fringes, making it difficult to distinguish the real microstructure. In contrast, the TEM and STEM bright field images of the flash polished sample are free of any of the artefacts, revealing the true microstructure of the unirradiated steel. Additionally, we also found flash polishing yielded a FIB sample with more than a year-long shelf life, likely due to the formation of a passivation layer during the electrochemical reaction. Follow-on work with neutron and ion irradiated samples revealed similar success, giving confidence that a combination of FIB milling and flash electropolishing can produce artefact free TEM foils. 


\section{References:}

[1] A Aitkaliyeva, JW Madden, BD Miller, JI Cole, Journal of Nuclear Materials 459 (2015) 241-246.

[2] M. Presley, Ph.D Dissertation, Ohio State University (2016).

[3] Y Huang, H Zhang, M.A. Auger, Z. Hong, H. Ning, M.J. Gorley, P.S. Grant, M.J. Reece, H. Yan, Journal of Nuclear Materials 487 (2017) 433-442.

[4] The authors acknowledge funding from the U.S. Department of Energy Office of Nuclear Energy's Nuclear Energy Enabling Technologies program project CFA 16-10570.

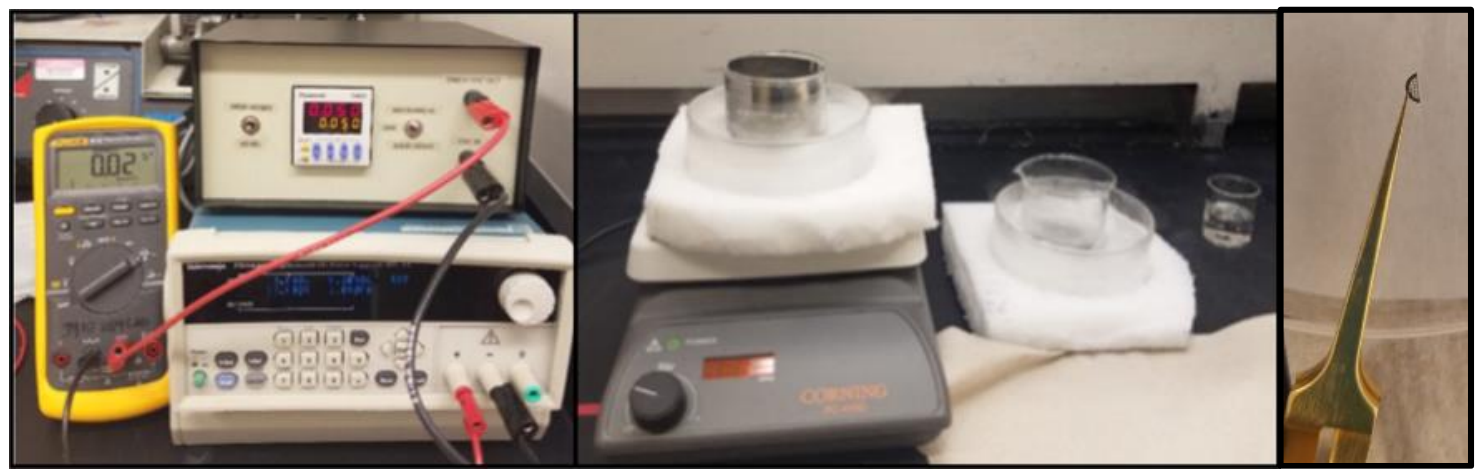

Figure 1. A simple beaker polishing setup is shown for flash polishing FIB samples mounted on a Au coated grid. The grid is held by the tweezers placed inside a plastic cap; the cap is inserted over the beaker.

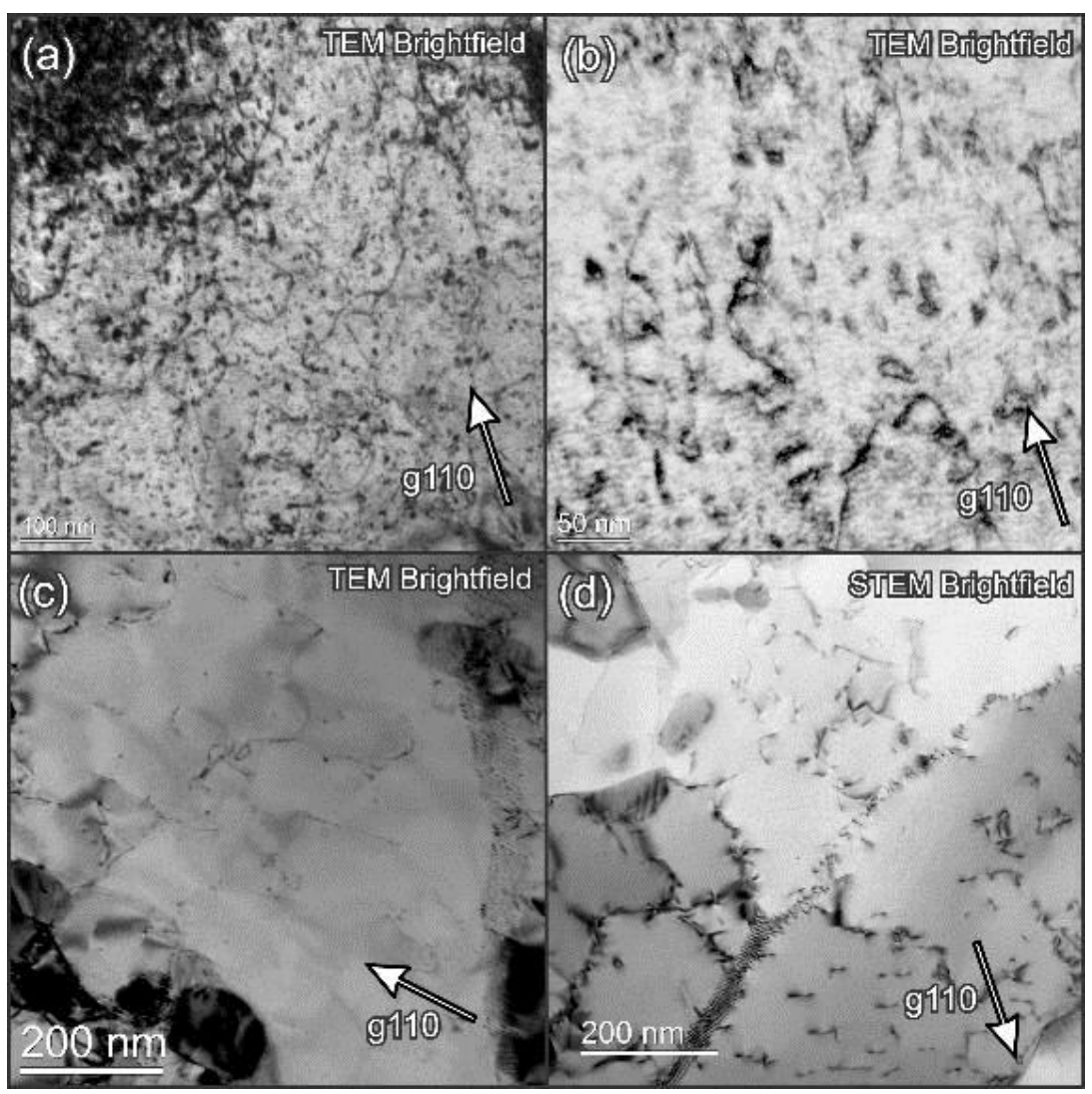

Figure 2. TEM bright field images shown in (a) and (b) highlight the "black spot" damage and moiré fringes present in the as-FIB sample. Neither of these two artefacts are present after flash polishing, as shown in the bright field images in (c) and (d). 